\title{
Computed Tomography Angiography (CTA) Features of Aortic Floating Thrombus: Five Case Reports and Literature Review
}

\author{
Bo Zhang ${ }^{1}$, Ji Zhang ${ }^{1,{ }^{*}}$, Lin-Yun $\mathrm{Wu}^{2}$, Zhong Wei Tian ${ }^{1}$, Hong $\mathrm{Yu}^{3}$ and Xiu-Ping Wang ${ }^{1}$ \\ ${ }^{1}$ Department of Radiology, Taizhou People’s Hospital, Taizhou, Jiangsu Province, China \\ ${ }^{2}$ Taizhou Polytechnic College, Taizhou, Jiangsu Province, China \\ ${ }^{3}$ Department of Radiology, First Affiliated Hospital of Dalian Medical University, Dalian, Liaoning Province, China \\ "Corresponding author: Department of Radiology, Taizhou People's Hospital, Taizhou, Jiangsu Province, China. Email: zj00151@126.com \\ Received 2021 May 11; Revised 2021 October 09; Accepted 2021 October 11.
}

\begin{abstract}
Aortic floating thrombus (AFT) is a rare disease, which is often misdiagnosed as a tumor or another disease. Here, we report five cases of AFT, confirmed by computed tomography angiography (CTA). The patients' laboratory biomarkers, clinical treatment, dynamic changes, and CTA features, including the AFT location, morphology, size, and aortic segment involvement, were retrospectively analyzed. CTA was the main imaging modality for detecting AFT, as it could depict lesions and determine the therapeutic effects accurately. Overall, the therapeutic strategy should be selected individually, depending on the patient's physical health; conservative medication use is also recommended.
\end{abstract}

Keywords: Ascending Aorta, Floating Thrombus, CTA

\section{Introduction}

Aortic mural thrombus (AMT), which is commonly caused by severe aortic atherosclerosis, aortic aneurysm, aortic dissection, and Takayasu's arteritis, is often attached to the inner wall of the aorta. As the distal end of AMT can float freely in the blood flow, the term "aortic floating thrombus" (AFT) has been widely used to describe it. Generally, AFT is described as a specific type of AMT. While AMT is more common, AFT is a very rare disease with an unclear etiology.

A hypercoagulable state and aortic disease are the main etiologies of AFT $(1,2)$. In computed tomography angiography (CTA) of the aorta, AFT has been mostly found in the thoracic and abdominal aortas, while it is rarely found in the ascending aorta. Here, we report five cases of AFT in the ascending aorta and present the CTA findings, clinical treatment, and follow-up changes in the patients.

\section{Case Presentation}

\subsection{Case 1}

A 68-year-old male patient was admitted to the hospital because of recurrent heart palpitations for three years, which had aggravated for one month. Physical examinations showed no obvious abnormalities. The D-dimer level was 2,657 ug/L (normal level < $500 \mathrm{ug} / \mathrm{L}$ ). The results of CTA indicated AFT (Figure 1A-C). The break-off risk ratio (boRR) was estimated at 2.01, according to Yang's formula (boRR $=$ length of the free portion/length of the attached portion) (3). The patient was transferred to the cardiovascular surgery department for surgical treatment. The ascending aorta was dissected, and a red protuberant mass with a wide pedicle was found; it was tough, smooth, and complete without capsules (indistinct from the aortic intima) (Figure 1D).

The gross examination revealed that the tumor was red and smooth with a hard surface (approximate length, 5.8 $\mathrm{cm}$ ) (Figure 1E). Microscopically, fibrin formed a grid-like, layered pattern, filled with blood cells, most of which were red blood cells and evenly distributed white blood cells (Figure 1F). A final diagnosis of thrombus was established. One week after surgery, the patient was treated and discharged from the hospital. Following discharge, the patient was prescribed aspirin and clopidogrel antiplatelet therapy. Besides, CTA was repeated at 12 months postoperatively. There was no thrombosis in the ascending aorta, and the blood flow was normal.

\subsection{Case 2}

A 58-year-old male patient was admitted to the hospital because of chest tightness, lower back pain, and left lower limb pain for two hours. He had a history of hypertension for 15 years. Upon admission, he presented with acute chest 

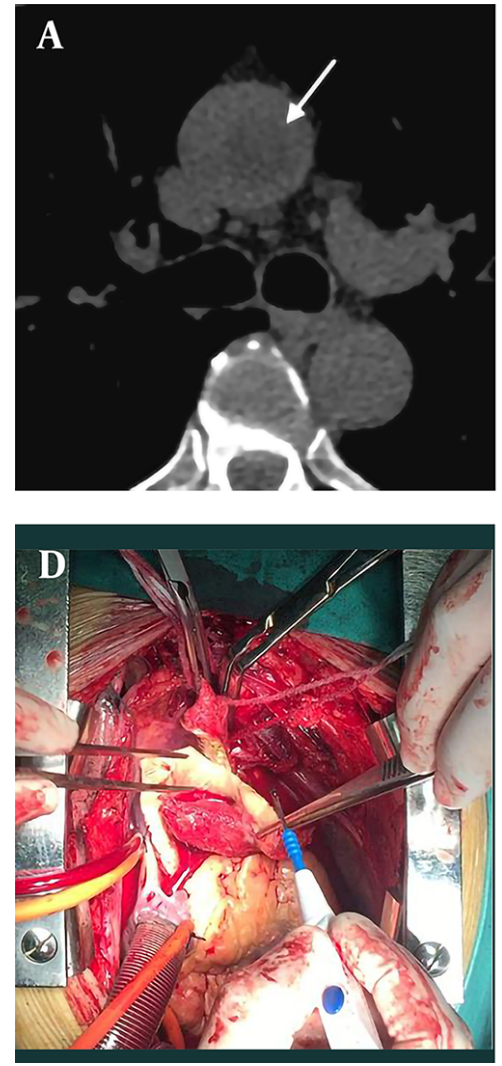
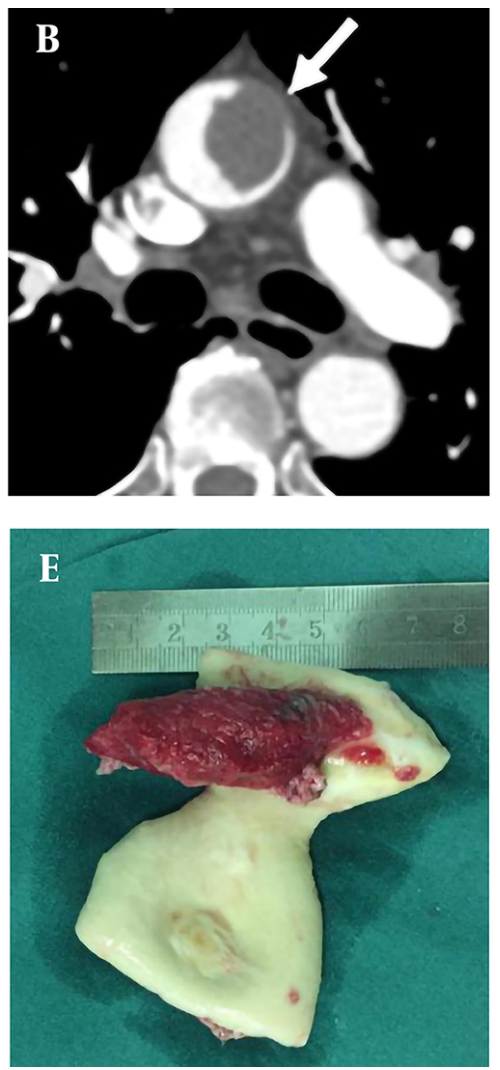
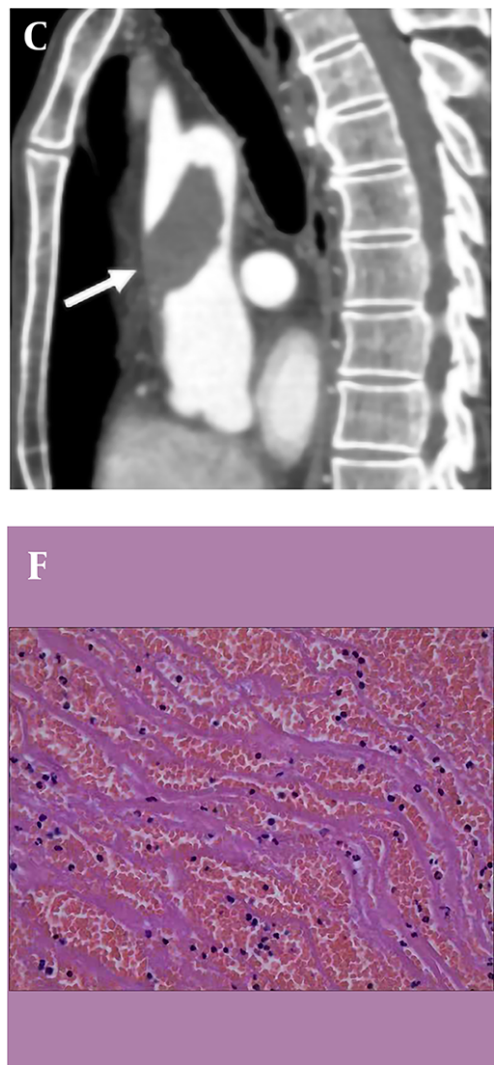

Figure 1. A, A 68-year-old male patient with heart palpitations for three years, aggravated for one month. CTA shows a slightly low-density filling defect in the ascending aorta (white arrow); B, Contrast enhancement of CTA indicates local thickening of the left anterior wall of the ascending aorta (white arrow). The filling defect size is approximately $1.5 \times 2.5 \mathrm{~cm}$; C, Multi-planar reconstruction indicates that the long axis of the lesion is along the direction of blood flow (white arrow). The proximal part is attached to the wall, and the distal part is free with a length of about $5.0 \mathrm{~cm}$; D, During surgery, the tumor was tough, smooth, and complete without capsules; E, The gross pathological specimens show a red protuberant mass with a broad pedicle, connected to the aortic wall; F, A diagnosis of thrombus is established (haematoxylin and eosin [H\&E] staining, $\times 400$ magnification).

pain and back pain; however, his vital signs were stable, and no obvious abnormality was found in the physical examination. The D-dimer level was 1,965 ug/L. Following admission, triple rule-out CTA and CTA examination of the left lower extremity showed no obvious abnormalities in the coronary or pulmonary arteries. However, the left wall of the ascending aorta was partially thickened with a filling defect (Figure $2 \mathrm{~A}$ and B). Local kidney infarction and occlusion of the left popliteal artery were also detected (Figure 2C and D). The boRR was measured to be 4.9 .

Under general anesthesia and cardiopulmonary bypass, the ascending aorta was excised, and artificial vascular replacement was performed. Meanwhile, a Fogarty arterial embolectomy catheter was used for the left lower extremity artery. After discharge, aspirin and clopidogrel were regularly used; no new thrombosis was detected after 24 months.

\subsection{Case 3}

A 57-year-old male patient was hospitalized for sudden chest pain for four hours while driving. He had a history of hypertension for 15 years. The level of D-dimer was 2,143 ug/L. The CTA examination showed that the anterior wall of the ascending aorta was thickened, with a filling defect of $3.3 \times 1.7 \mathrm{~cm}$ approximately (Figure 3 ). A floating thrombus was suggested in the anterior wall of the ascending aorta; the boRR was measured to be 5.4. Because of the high risk of arterial thrombosis, surgical treatment was recommended. The ascending aorta was resected, and the artificial vessel was replaced. Anticoagulants were used by the patient regularly. During the 36-month follow-up, the results of CTA were normal.

\subsection{Case 4}

A 71-year-old male patient was hospitalized for recurrent chest tightness for one month. He had a 20-year 

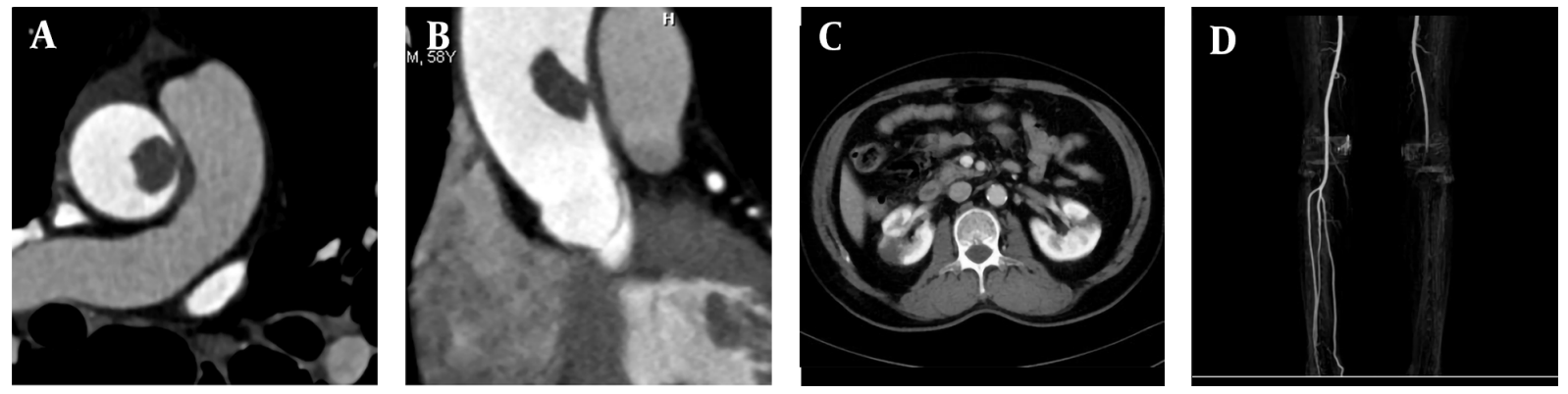

Figure 2. A, A 58-year-old male patient with chest tightness, lower back pain, and left lower limb pain for two hours. CTA shows local thickening of the right wall of the ascending aorta with a filling defect (approximate size, $1.2 \times 1.5 \mathrm{~cm}$ ); B, Multi-planar reconstruction indicates that the long axis of the lesion is in the direction of blood flow. The proximal segment is attached to the wall, while the distal segment is free; C, Contrast-enhanced CT cross-section shows bilateral segmental renal infarction; D, Maximum density projection of CTA for both lower limbs indicates the left popliteal artery embolism; the distal vessels are not clear.
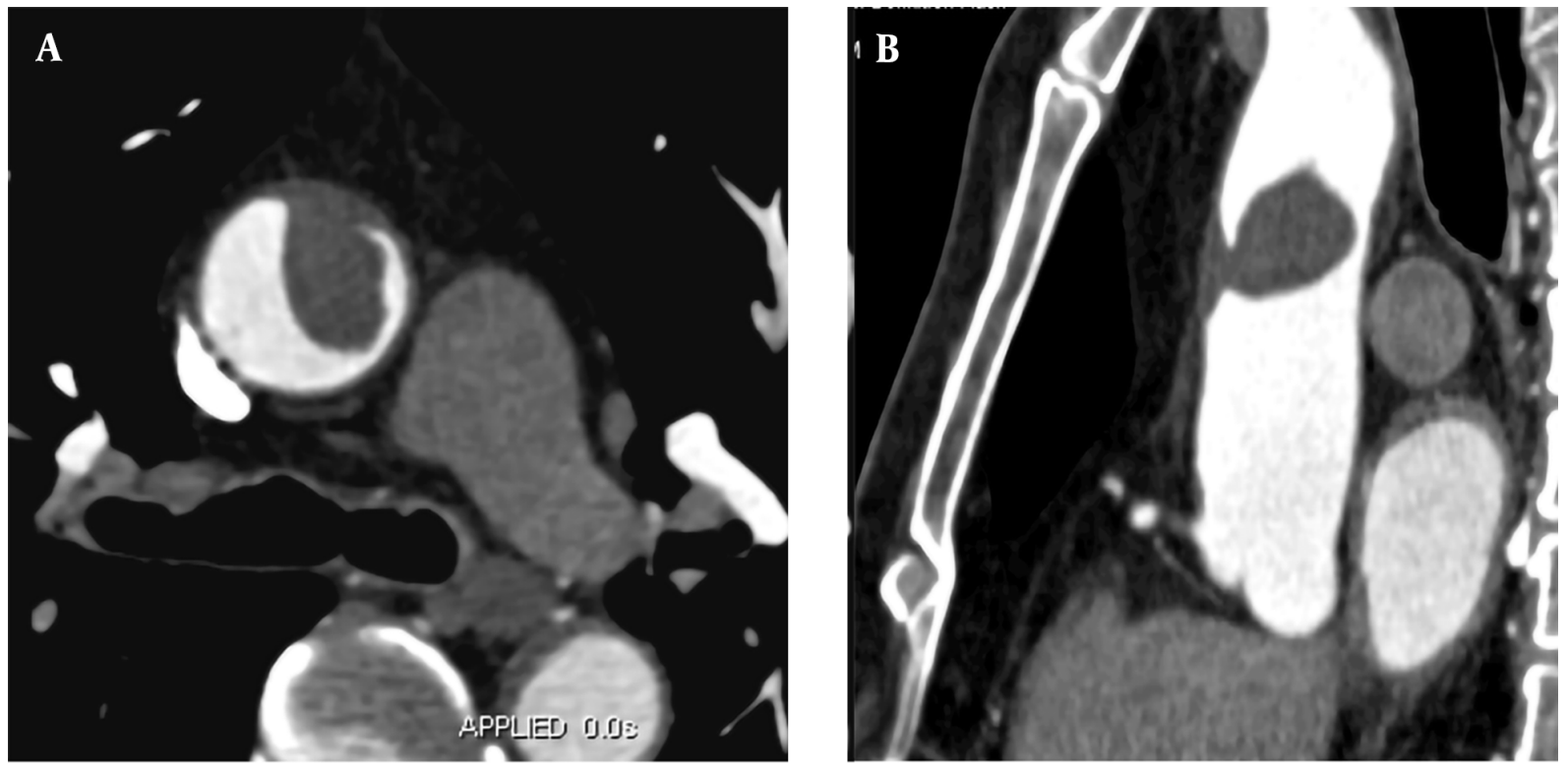

Figure 3. A, A 57-year-old male patient experiencing sudden chest pain for four hours while driving. CTA indicates local thickening of the anterior wall of the ascending aorta with a $3.3 \times 1.7 \mathrm{~cm}$ filling defect. B, Multi-planar reconstruction of CTA reveals that the long axis of the lesion is in the direction of blood flow, with the proximal part attached to the vascular wall and the distal part floating free.

history of hypertension. His blood pressure was 190/100 mmHg. The level of D-dimer was $1,869 \mathrm{ug} / \mathrm{L}$. The CTA examinations of coronary and pulmonary arteries showed no abnormal findings. The results of CTA showed two filling defects in the anterior and posterior walls of the ascending aorta (Figure 4A); the boRR was 2.2 and 5.5, respectively. The patient refused surgical treatment and was transferred to an advanced specialty hospital for treatment. Regular anticoagulation and thrombolysis treatment with oral rivaroxaban were performed for the patient after one month. The ascending aorta wall was smooth, and no filling defect was found in the lumen (Figure 4B). Conservative treatment was considered suitable for the patient. After 18 months, there was no new thrombosis on CTA.

\subsection{Case 5}

A 72-year-old male patient was admitted to the hospital for recurrent chest tightness for two months. He had no significant medical history or obvious abnormalities in the physical examination. The D-dimer level was $300 \mathrm{ug} / \mathrm{L}$ (normal level $<500$ ug/L). Following admission, CTA showed no obvious abnormalities in the coronary arteries. However, the left wall of the ascending aorta was locally thickened, and a papillary filling defect was found (Figure 5); the boRR 

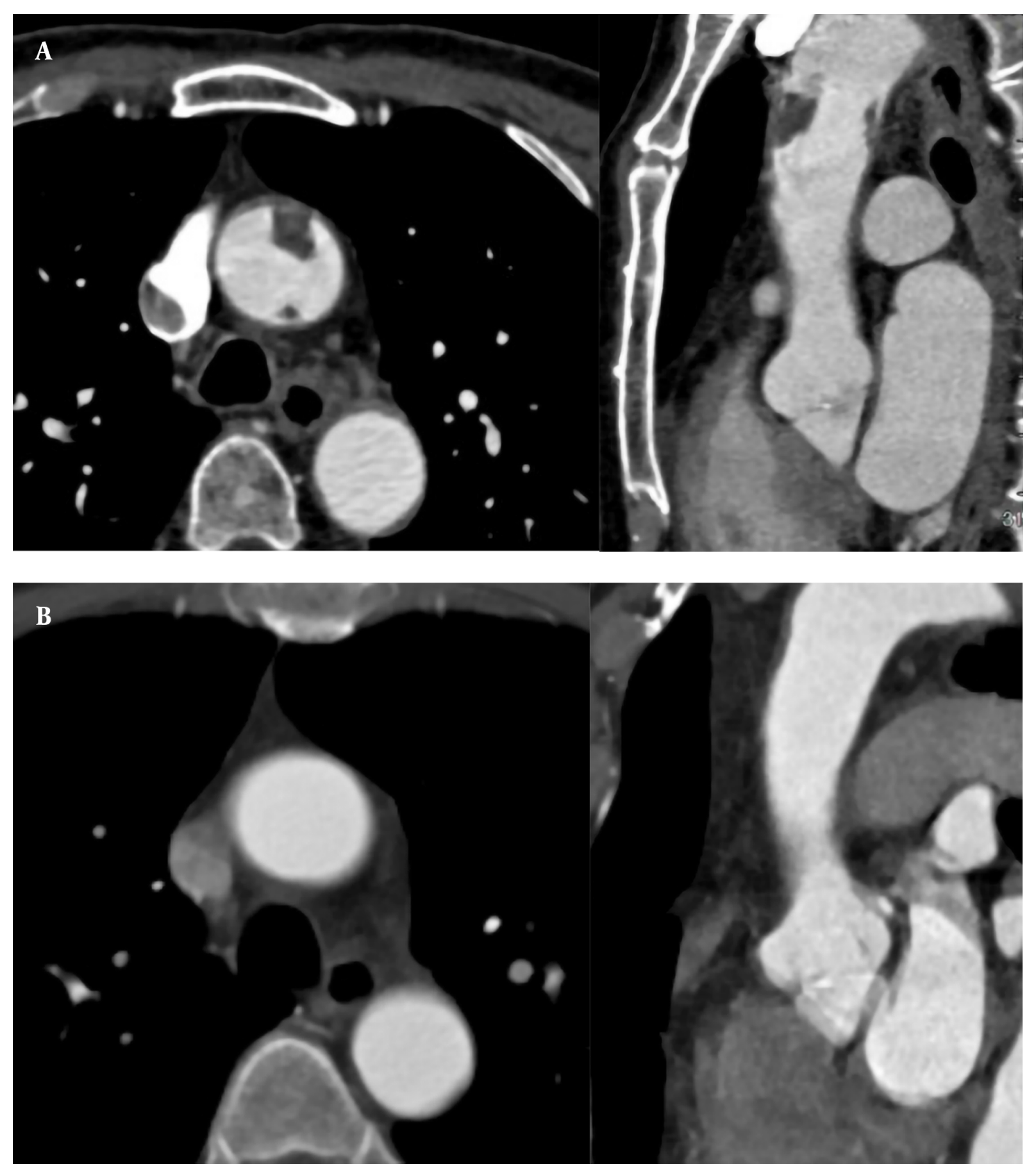

Figure 4. A, A 71-year-old male patient with recurrent chest tightness for one month. CTA indicates filling defects in the anterior and posterior walls of the ascending aorta (1.4 $\times 0.9$ and $0.3 \times 0.5 \mathrm{~cm}$, respectively). Multi-planar reconstruction shows that the long axis of the two lesions is in the direction of blood flow; B, Thrombus disappeared one month after thrombolytic therapy. 
was measured to be 2.9. AFT disappeared after two months of regular anticoagulation and thrombolysis with rivaroxaban.

The clinical characteristics and follow-up data of the five cases examined in this study are presented in Table 1. The detailed CTA imaging findings are also shown in Table 2.

\section{Discussion}

AMT can be divided into fixed and pedicled types according to its morphology. Because the distal ends of pedicled thrombi may seem to float in the blood flow on ultrasound, computed tomography (CT), and dynamic magnetic resonance imaging (MRI), they are known as floating thrombi. Although the incidence and distribution of aortic thrombosis remain unclear, they are often associated with aortic aneurysms; in other words, non-aortic aneurysm thrombosis is rare. In this regard, Machleder et al. (4) reported that the incidence of non-aortic aneurysm thrombosis was $0.45 \%(45 / 10,671)$ in autopsy cases.

A floating thrombus is more likely to cause severe peripheral vascular embolism compared to a fixed thrombus, such as lower extremity artery, superior mesenteric artery, renal artery splenic artery and upper limb artery. Intracranial and coronary arteries are rarely involved, but the prognosis is poor once it happened. Intracranial and coronary arteries are rarely involved, although the prognosis is poor. The incidence rate of AFT is low, and its clinical diagnosis is easy to miss. Currently, with the extensive application of CTA, it is possible to detect AFT, which is of great significance for the etiology, diagnosis, treatment, and prognosis of the disease.

High blood pressure and high blood flow in a normal aorta are not usually associated with thrombosis; higher blood pressure and higher blood flow in the ascending aorta make thrombosis even rarer. In this regard, a metaanalysis by Fayad et al. (5) showed that the incidence of thrombosis was $11.6 \%$ in the ascending aorta, while it often exceeded 30\% in the aortic arch and descending aorta. The predisposing conditions for thrombosis commonly include intimal injury to the vessel, vascular state change, and abnormal blood coagulation. Although the etiology of AFT is unknown, it may be related to aortic atherosclerosis, aortic aneurysm, hematological diseases, malignant tumors, anticancer therapy, hormone therapy, connective tissue diseases, iatrogenic conditions, trauma, hyperhomocysteinemia due to heavy drinking, and a hypercoagulable state due to pregnancy $(1,2,6,7)$.

In the present study, local wall thickening, with or without calcification was found in four patients in a hypercoagulable state, indicating that aortic wall atheroscle- rosis and a hypercoagulable state are the main causes of a floating thrombus in the ascending aorta. Besides, other studies have reported a floating thrombus of the ascending aorta in a normal aortic wall and a nonhypercoagulable state. AFT is commonly located at the junction of the aortic sinus and the sinus tube; its special anatomical structure, where turbulence is easy to form, may result in a transient local hypercoagulable state $(8,9)$.

It is generally believed that the biological characteristics of the ascending aorta determine the target sites for thrombosis. In this regard, von Knobelsdorff-Brenkenhoff et al. (10) reported the specificity of blood flow in the ascending aorta, as the blood flow velocity near the posterior wall of the ascending aorta is slow with a low shear force, while near the right anterior wall, it is fast with a high shear force. A low shear force is related to a high risk of plaque progression caused by inflammation (11), while a high shear force is associated with atherosclerosis. The morphology of the ascending aorta often changes with advancing age; therefore, change of shear force in the aortic wall is more likely to occur in the right posterior wall. Therefore, from a biomechanical point of view, the right posterior wall is the most vulnerable site for thrombosis. However, according to our literature review, floating thrombi in the ascending aorta are randomly distributed.

In this study, two out of the six AFTs were located in the anterior wall, one in the left anterior wall, two in the left wall, one in the posterior wall, and none in the right posterior wall. Besides biomechanics, there may be other factors related to the ascending aorta thrombosis. AFT was displayed as a low-density filling defect in the aortic lumen on CTA images. It could be spherical, cylindrical, flaky, or papillary in shape, connected to the aortic wall with a narrow base or pedicle. Most AFTs were in the direction of blood flow and floated with the heartbeat without contrast enhancement. In this group, five large AFTs were found in the direction of blood flow, and one smaller AFT was found perpendicular to the aortic wall.

According to Yang's boRR formula (3), the risk coefficient of six AFTs was in the range of 2.01-5.54. Theoretically, the higher the boRR is, the greater the possibility of thrombus shedding will be. However, boRR data has not been supported in large samples, and its statistical significance remains unclear. According to our review of the literature, AFT does not break off easily mostly. Some AFTs are still firmly connected to the aortic wall, although peripheral vascular embolism happened.

Yang et al. (3) reported two cases of AFT with partial splenic infarction and segmental embolism of the superior mesenteric artery. The end of AFT was bifurcated, which might be related to the emergence of a new growth endpoint after the local portions of thrombus break off. 

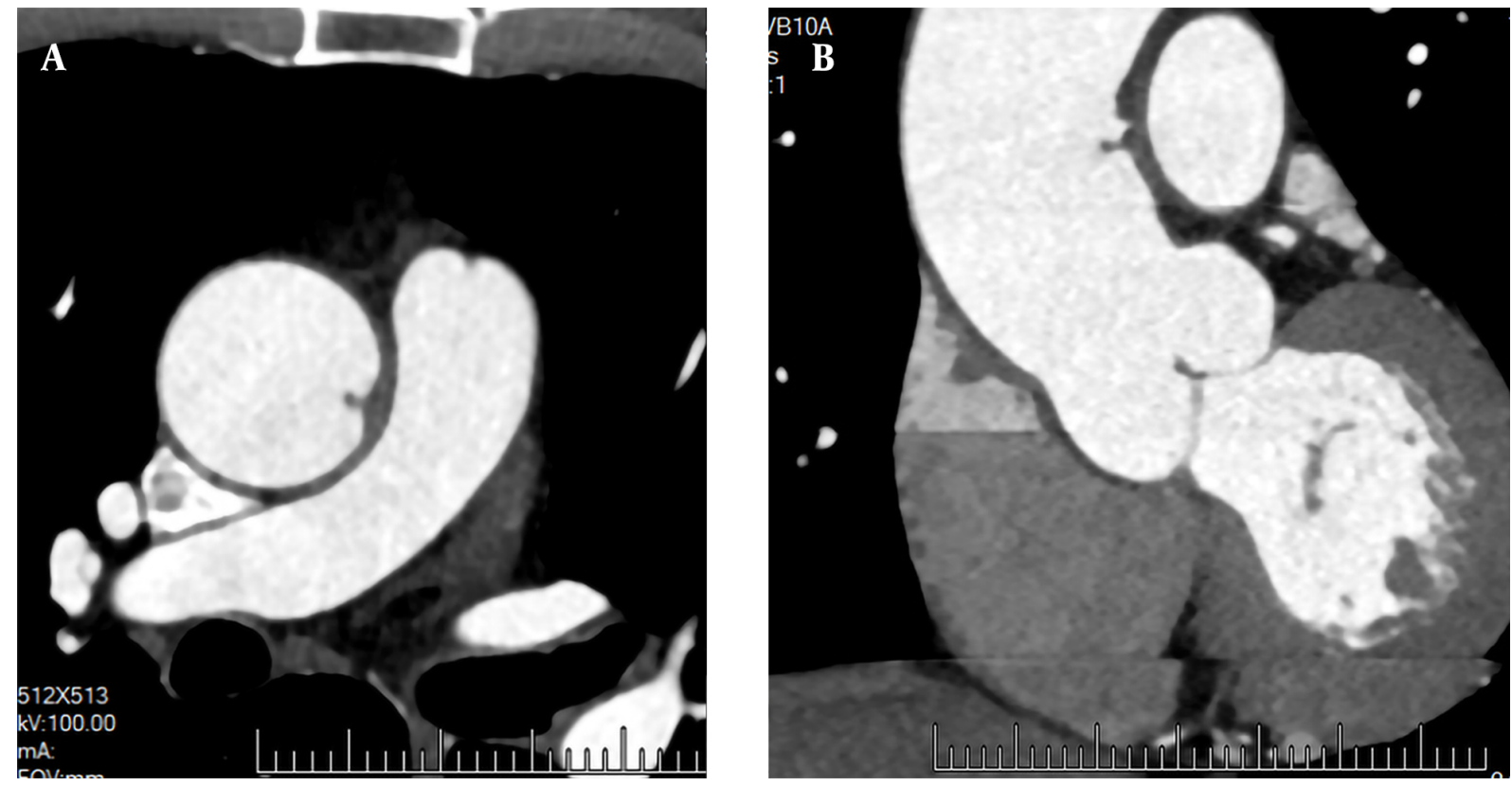

Figure 5. A 72-year-old male patient with recurrent chest tightness for two months. CTA cross-section (A) and multi-planar reconstruction (B) indicate a papillary filling defect in the left wall of the ascending aorta, perpendicular to the aortic wall.

\begin{tabular}{|c|c|c|c|c|c|c|c|}
\hline Case & Age $(y)$ & Sex & Chief complaint & History & $\begin{array}{l}\text { D-dimer level } \\
(<500 \mathrm{ug} / \mathrm{L})\end{array}$ & Treatment & Prognosis \\
\hline $\mathbf{1}$ & 68 & Male & $\begin{array}{l}\text { Heart palpitations for } \\
\text { three years, aggravated for } \\
\text { one month }\end{array}$ & None & 2,657 & $\begin{array}{l}\text { Ascending aorta resection and } \\
\text { artificial vascular replacement }\end{array}$ & Good \\
\hline 2 & 58 & Male & $\begin{array}{l}\text { Chest tightness, low back } \\
\text { pain, and left lower limb } \\
\text { pain for two hours }\end{array}$ & $\begin{array}{l}\text { Hypertension and } \\
\text { diabetes for } 12 \text { years }\end{array}$ & 1,965 & $\begin{array}{l}\text { Ascending aorta resection, artificial } \\
\text { vessel replacement, and Fogarty } \\
\text { catheter embolectomy of the left } \\
\text { lower extremity artery }\end{array}$ & Good \\
\hline 3 & 57 & Male & $\begin{array}{l}\text { Sudden chest pain for four } \\
\text { hours }\end{array}$ & $\begin{array}{l}\text { Hypertension for } 15 \\
\text { years }\end{array}$ & 2,143 & $\begin{array}{l}\text { Ascending aorta resection and } \\
\text { artificial vascular replacement }\end{array}$ & Good \\
\hline 4 & 71 & Male & $\begin{array}{l}\text { Recurrent chest tightness } \\
\text { and palpitations for one } \\
\text { month }\end{array}$ & $\begin{array}{l}\text { Hypertension for } 20 \\
\text { years }\end{array}$ & 1,869 & $\begin{array}{l}\text { Anticoagulant therapy for resolving } \\
\text { thrombus }\end{array}$ & Good \\
\hline 5 & 72 & Male & $\begin{array}{l}\text { Recurrent chest tightness } \\
\text { for two months }\end{array}$ & None & 300 & $\begin{array}{l}\text { Anticoagulant therapy for resolving } \\
\text { thrombus }\end{array}$ & Good \\
\hline
\end{tabular}

Therefore, thrombus shedding not only depends on the overall thrombus activity, size, morphology, and boRR, but is also related to the duration of thrombosis formation and the local blood flow, because a fresh thrombus is more likely to break off in a high aortic blood flow. Besides, one patient with a boRR of 4.9 showed bilateral lower back pain and left lower limb pain.

The CT examination indicated localized renal infarction and popliteal artery embolism in the left lower extremity; aortic lumen thrombosis still existed. The cause of peripheral vascular embolism may be local intraluminal turbulence and a hypercoagulable state caused by thrombus, along with a local fresh thrombus on the entire thrombus surface, broken off due to high blood flow. Besides, thrombus can lead to iatrogenic interventions. As reported in the literature, when a thrombus is fresh, it breaks off easily in the blood flow (12).

The clinical manifestations of AFT are non-specific and usually associated with embolism of the central nervous system, coronary arteries, mesenteric vessels, and arteries in the extremities due to thrombus shedding, which is often treated following chest pain and abdominal pain 


\begin{tabular}{|c|c|c|c|c|c|c|c|}
\hline Case & Number & Location & Shape & boRR & Affected aorta & Floating direction & $\begin{array}{l}\text { Complicated } \\
\text { embolism }\end{array}$ \\
\hline 1 (Figure 1) & 1 & Left anterior wall & Cylindrical & 2.01 & $\begin{array}{l}\text { Local aortic wall } \\
\text { thickening }\end{array}$ & Along the blood flow & None \\
\hline 2 (Figure 2) & 1 & Left lateral wall & Elliptical & 4.9 & $\begin{array}{l}\text { Local aortic wall } \\
\text { thickening }\end{array}$ & Along the blood flow & $\begin{array}{l}\text { Infarction of both } \\
\text { kidneys and the left } \\
\text { popliteal artery } \\
\text { embolism }\end{array}$ \\
\hline 3 (Figure 3) & 1 & Anterior wall & Elliptical & 5.4 & $\begin{array}{l}\text { Local aortic wall } \\
\text { thickening }\end{array}$ & Along the blood flow & None \\
\hline 4 (Figure 4) & 2 & $\begin{array}{c}\text { Anterior wall and } \\
\text { posterior wall }\end{array}$ & Schistose & $2.22 \& 5.5$ & $\begin{array}{l}\text { Local aortic wall } \\
\text { thickening and } \\
\text { calcification }\end{array}$ & Along the blood flow & None \\
\hline 5 (Figure 5) & 1 & Left lateral wall & Papillary & 2.9 & $\begin{array}{l}\text { Local aortic wall } \\
\text { thickening }\end{array}$ & Vertical & None \\
\hline
\end{tabular}

Abbreviations: boRR, break-off risk ratio; CTA, computed tomography angiography.

of unknown origins. In our group of five patients, embolism caused by fresh thrombus breaking off from the surface of AFT was found in one patient with sudden bilateral lower back pain and left lower limb pain. One patient was diagnosed with acute chest pain, and AFT was found in the aortic CTA; however, no abnormality was found in the coronary artery CTA or pulmonary artery CTA. It was assumed that the fresh thrombus formed on the AFT surface led to the embolization of small branches of the thoracic aorta. Three cases were discovered by accident during medical treatment for other reasons. Therefore, AFT should be taken into consideration in patients with a hypercoagulable state and unexplained chest pain and abdominal pain.

On CTA images, AFT emerged as a partially wallattached, partially free, or papillary filling defect in the aortic lumen, floating along the blood flow in multi-phase cine images (systolic and diastolic phases); it was not difficult to diagnose AFT, and the main differential diagnosis was aortic intimal sarcoma. Clinically, patients may be treated for pain caused by vascular embolism. AFT is generally connected to the aorta with a narrow base or pedicle in images with smooth edges, without contrast enhancement. Aortic endothelial sarcoma is usually connected to the aorta through a broad base with uneven margins, and intimal sarcoma is detected after enhancement (13).

Diagnostic imaging modalities for AFT usually include transesophageal echocardiography (TEE), CTA, and MRI, which can accurately diagnose AFT and provide real-time dynamic images more conveniently. However, the connection between the ascending aorta and the aortic arch is disturbed by the trachea, and the field of view is poor; therefore, lesions may be missed in the corresponding areas. CTA of the aorta can be used to accurately diagnose AFT, evaluate the risk of break-off, and understand whether there is embolism in the aortic branch and whether it is combined with abdominal organ infarction; this can be helpful for follow-up after treatment.

Moreover, dynamic cine CT imaging can be used to observe the dynamics of thrombus formation and show the floating state of AFT in the lumen more accurately. Cine MRI imaging not only can present the floating state of the free portion of AFT dynamically, but also can identify whether the lesion in the aortic lumen is neoplastic. However, the examination time is long, and the cost is high. Therefore, CTA is the first choice for the preoperative diagnosis and postoperative follow-up of AFT.

Due to the rarity of AFT, there is no standardized treatment plan or clinical guidelines for this disease. The treatment plan is mainly based on the patient's condition and the clinician's experience and include drug therapy, interventional therapy, and open surgery. Two patients in this group were treated with rivaroxaban, in whom thrombi disappeared, and no embolism occurred during the disease. Three patients in this group underwent surgical resection and artificial vascular replacement, after which they used anticoagulants regularly and had a good prognosis.

Several studies have recommended drug therapy as the first choice of treatment, especially for asymptomatic cases $(3,14)$. However, there is no unified standard for the optimal drug, dosage, or duration of anticoagulant therapy. Ito et al. (15) reported rapid thrombolysis with a plasminogen activator. However, thrombolytic therapy could increase the incidence of embolism events and bleeding complications; warfarin and rivaroxaban were also used in case reports of complete thrombolysis of the ascending aorta (16-18). The risk of thrombus break-off is lower during an interventional procedure compared to an open surgery. Since the lesion can easily break off during surgery, a filter 
screen on the distal side of the lesion can prevent remote embolism caused by thrombus shedding.

Endovascular resection with stent implantation is suitable for patients with ineffective drug treatments and poor general conditions, making them unsuitable candidates for open surgery. However, there is a certain risk of recurrence, and the long-term efficacy of treatment remains to be investigated. Open surgery is generally suitable for patients with a high preoperative suspicion of malignant lesions, cases of large lesions with a high risk of break-off, and recurrent thrombosis cases. Postoperative pathological findings of lesions and the surrounding tube walls are of great significance for determining the cause of lesions and future treatment plans $(19,20)$. Overall, long-term individualized drug therapy and follow-up are particularly important for improving prognosis.

In conclusion, AFT is a rare aortic disease, which may lead to distant vascular embolism and organ infarction due to thrombus shedding. AFT can occur in patients with coagulopathy, primary aortic lesions, and regional aortic blood flow abnormalities due to various causes. When patients with these underlying diseases have symptoms of unexplained chest pain and abdominal pain, coagulation function test and aortic CTA need to be performed. Once AFT is detected, better therapeutic outcomes can be achieved through timely and effective individualized treatments with improved patient prognosis; if necessary, CTA review can be used to evaluate the efficacy of treatment and complications.

\section{Footnotes}

Authors' Contribution: Bo Zhang reviewed the literature and drafted the manuscript. Wei-Zhong Tian supervised and reviewed the manuscript. Ji Zhang prepared the images and proofread the manuscript. Xiu-Ping Wang and Chong-Fu Jia were responsible for collecting the data of the five patients. Lin-Yun Wu revised the manuscript for English grammar.

Conflict of Interests: The authors declare no conflict of interest.

Ethical Approval: The informed consent of the 5 patients was obtained mentioned in this article.

Funding/Support: This work was supported by Jiangsu Province's 333 High-Level Talent Project (grant number: BRA2020193), Jiangsu Provincial Medical Youth Talent (grant number: QNRC2016509), and Jiangsu Provincial High-Level Talent Project (grant number: LGY2018032).

Informed Consent: Informed consent was obtained from five patients included in this study.

\section{References}

1. Weiss S, Buhlmann R, von Allmen RS, Makaloski V, Carrel TP, Schmidli J, et al. Management of floating thrombus in the aortic arch. J Thorac Cardiovasc Surg. 2016;152(3):810-7. doi: 10.1016/j.jtcvs.2016.03.078. [PubMed: 27160939].

2. Marin-Acevedo JA, Koop AH, Diaz-Gomez JL, Guru PK. Nonatherosclerotic aortic mural thrombus: A rare source of embolism. BMJ Case Rep. 2017;2017. doi: 10.1136/bcr-2017-220592. [PubMed: 28765187]. [PubMed Central: PMC5623256].

3. Yang S, Yu J, Zeng W, Yang L, Teng L, Cui Y, et al. Aortic floating thrombus detected by computed tomography angiography incidentally: Five cases and a literature review. J Thorac Cardiovasc Surg. 2017;153(4):791-803. doi: 10.1016/j.jtcvs.2016.12.015. [PubMed: 28088428].

4. Machleder HI, Takiff H, Lois JF, Holburt E. Aortic mural thrombus: An occult source of arterial thromboembolism. JVasc Surg.1986;4(5):4738. [PubMed: 3773130].

5. Fayad ZY, Semaan E, Fahoum B, Briggs M, Tortolani A, D’Ayala M. Aortic mural thrombus in the normal or minimally atherosclerotic aorta. Ann Vasc Surg. 2013;27(3):282-90. doi: 10.1016/j.avsg.2012.03.011. [PubMed: 22929167].

6. Sawada T, Shimokawa T. Giant thrombus in the ascending aorta that caused systemic embolism. Interact Cardiovasc Thorac Surg. 2011;12(6):1048-50. doi: 10.1510/icvts.2011.266445. [PubMed: 21422157].

7. Goueffic Y, Chaillou P, Pillet JC, Duveau D, Patra P. Surgical treatment of nonaneurysmal aortic arch lesions in patients with systemic embolization. J Vasc Surg. 2002;36(6):1186-93. doi: 10.1067/mva.2002.128933. [PubMed: 12469050].

8. Bruno P, Massetti M, Babatasi G, Khayat A. Catastrophic consequences of a free floating thrombus in ascending aorta. Eur J Cardiothorac Surg. 2001;19(1):99-101. doi: 10.1016/s1010-7940(00)00600-x. [PubMed: 11163572].

9. Ozaki N, Yuji D, Sato M, Inoue K, Wakita N. A floating thrombus in the ascending aorta complicated by acute myocardial infarction. Gen Thorac Cardiovasc Surg. 2017;65(4):213-5. doi:10.1007/s11748-015-0619-7. [PubMed: 26728032]

10. von Knobelsdorff-Brenkenhoff F, Karunaharamoorthy A, Trauzeddel RF, Barker AJ, Blaszczyk E, Markl M, et al. Evaluation of Aortic Blood Flow and Wall Shear Stress in Aortic Stenosis and Its Association With Left Ventricular Remodeling. Circ Cardiovasc Imaging. 2016;9(3). e004038. doi: 10.1161/CIRCIMAGING.115.004038. [PubMed: 26917824]. [PubMed Central: PMC4772425].

11. Gitsioudis G, Chatzizisis YS, Wolf P, Missiou A, Antoniadis AP, Mitsouras D, et al. Combined non-invasive assessment of endothelial shear stress and molecular imaging of inflammation for the prediction of inflamed plaque in hyperlipidaemic rabbit aortas. Eur Heart J Cardiovasc Imaging. 2017;18(1):19-30. doi: 10.1093/ehjci/jew048. [PubMed: 27013245]. [PubMed Central: PMC5217740].

12. Endo H, Ishii H, Tsuchiya H, Takahashi $Y$, Shimoyamada H, Isomura A, et al. Pathologic Features of Lone Aortic Mobile Thrombus in the Ascending Aorta. Ann Thorac Surg. 2016;102(4):e313-5. doi: 10.1016/j.athoracsur.2016.03.107. [PubMed: 27645970].

13. Toyama M, Nakayama M, Hasegawa M, Yuasa T, Sato B, Ohno O. Direct oral anticoagulant therapy as an alternative to surgery for the treatment of a patient with a floating thrombus in the ascending aorta and pulmonary embolism. J Vasc Surg Cases Innov Tech. 2018;4(2):1702. doi: 10.1016/j.jvscit.2018.03.002. [PubMed: 29942913]. [PubMed Central: PMC6012988].

14. Nakajima M, Tsuchiya K, Honda Y, Koshiyama H, Kobayashi T. Acute pulmonary embolism after cerebral infarction associated with a mobile thrombus in the ascending aorta. Gen Thorac Cardiovasc Surg. 2009;57(12):654-6. doi: 10.1007/s11748-009-0444-y. [PubMed: 20013100]. 
15. Ito H, Takahashi K, Sasaki H, Akiho H, Katahira Y, Saito H, et al. Large thrombus in the ascending aorta successfully treated by thrombolysis-an unusual cause of acute massive myocardial infarction. Jpn Circ J. 2001;65(6):572-4. doi: 10.1253/jcj.65.572. [PubMed: 11407743].

16. Rancic Z, Pfammatter T, Lachat M, Frauenfelder T, Veith FJ, Mayer D. Floating aortic arch thrombus involving the supraaortic trunks: successful treatment with supra-aortic debranching and antegrade endograft implantation. J Vasc Surg. 2009;50(5):1177-80. doi: 10.1016/j.jvs.2009.05.052. [PubMed: 19628356].

17. Kahlberg A, Montorfano M, Cambiaghi T, Bertoglio L, Melissano G, Chiesa R. Endovascular Stent-Grafting of the Ascending Aorta for Symptomatic Parietal Thrombus. J Endovasc Ther. 2016;23(6):969-72. doi: 10.1177/1526602816664877. [PubMed: 27530412].

18. Bowdish ME, Weaver FA, Liebman HA, Rowe VL, Hood DB. Anticoagulation is an effective treatment for aortic mural thrombi. J Vasc Surg. 2002;36(4):713-9. [PubMed: 12368731].

19. Scott DJ, White JM, Arthurs ZM. Endovascular management of a mobile thoracic aortic thrombus following recurrent distal thromboembolism: a case report and literature review. Vasc Endovascular Surg. 2014;48(3):246-50. doi: 10.1177/1538574413513845. [PubMed: 24347279].

20. Fukuhara S, Tyagi S, Clarke-Pearson E, Bernik T. Endovascular stent graft repair of thoracic aortic mural thrombus in a patient with polycythemia vera: A word of caution. Vascular. 2015;23(1):89-92. doi: 10.1177/1708538114525154. [PubMed: 24569113]. 